\title{
Study on Singular Spectrum Analysis as a data-driven technique for damage diagnosis. Comparison between time and frequency domain.
}

\author{
David Garcia ${ }^{1, *}$, and Irina Trendafilova ${ }^{1}$ \\ ${ }^{1}$ Mechanical and Aerospace Engineering, University of Strathclyde, 75 Montrose Street - Glasgow G1 1XJ - United Kingdom
}

\begin{abstract}
Vibration-based Structural Health Monitoring methodologies have been developed in many different applications with the aim of damage diagnosis. Recently, purely data-driven methods have been gained popularity because these methods do not assume any linearity or model in their analysis. Data-driven methods use the measured vibration signals as data-input to extract features that can conclude obtain useful information for the damage diagnosis. In this work a methodology based on Singular Spectrum Analysis (SSA) technique is presented which decomposes the measured vibration responses in a certain number of principal components which reveal the rotational patterns at any frequency in the motion. One of the steps of the methodology is to create a reference state where the observations can be compared for damage assessment. The data used to create the reference state determines how the information is represented in the reference state and therefore how meaningful and informative are the feature vectors for damage assessment. This study presents of the effect of the data representation considered on the creation of the reference state when the data is introduced in the time or frequency domain. The results obtained are different depending on the signal representation and hence they should have different interpretation when the state is created based on vibratory signals represented in the time or frequency domain.
\end{abstract}

\section{Introduction}

Structural health Monitoring (SHM) is a strategy that Engineers and Scientifics have been developing to understand and monitor the performance of structures for their design life expectation. SHM is considered a multidisciplinary technology with the goal of identifying and objectively quantify any occurrence regarding to the structural integrity. For decades monitoring the vibration responses on a structure have been used as an indicator of its condition based on assumption of any change due to a damage results in a more or less pronounced change of the dynamic behaviour [1], this is known as vibrationbased SHM (VSHM). Recently, purely data-driven methods have gained popularity because these methods do not assume any linearity or model in their analysis. Data-driven methods use the measured vibration signals as a data-input to subtract features that can conclude in useful information for damage diagnosis [2].

Over the last years, the philosophy of SHM has been defined in different manners. One of the most common one was introduced by Farrar et al. in [3]. The authors defined SHM procedure in four steps: (i) operation evaluation, (ii) data acquisition, (iii) feature extraction and (iv) decision making and classification. Each of these steps can devote a separate research to find useful and reliable techniques for developing successful SHM methods and address some of the fundamental axioms presented in [4].

The data-driven VSHM methodology based on singular spectrum analysis (SSA) presented by the authors in [5] is divided in four steps; data collection, creation of the reference state, feature extraction and damage assessment. The data used to create the reference state determines how much information is contained in the reference state and therefore how meaningful and informative are the feature vectors for damage assessment.

This manuscript presents the effects of the data representation (time or frequency domain) on the creation of the reference state. Although the idea is similar and the creation of the reference state follows the same steps for both cases of the data representation, the results obtained are different and therefore a different interpretation should be applied when the reference state is created based on vibration signals represented in the

\footnotetext{
* Corresponding author: david.garcia@strath.ac.uk
} 
time or frequency domain. In order to compare the effects on the creation of the reference state, the same vibration responses were considered in both analysis. The results were different for each case and hence they should have different interpretation.

\section{Damage assessment methodology - a short introduction.}

The methodology is based on singular spectrum analysis (SSA) [6] a technique which is a nonparametric method for data compression and information extraction. The technique finds linear combinations of the initial variables that describe and smooth the major trends and oscillations patterns in the vibration responses measured on the structure/system in consideration.

The steps considered in the methodology presented from the authors in [5] are data collection, creation of the reference state, feature extraction and damage assessment. The aim of this study is to find the differences when the reference state is constructed in the time or frequency domain. Therefore this will be the only step to be analysed in this manuscript.

Creation of the reference state: The main purpose of the methodology is to detect the differences between healthy and damaged structures. The reference state is generally constructed by signal vectors measured in the healthy/reference structure. This is the state where the newly observed signals, measured on the structure, will be compared to the reference state for making the decision whether the structure is healthy or damaged.

However the aim of this study is not to identify if a structure is healthy or damaged but how informative the reference state will be when the vector signals used to build the reference state, are considered/represented in the time or in the frequency domain. For this reason the reference state will be created first by signal vectors in the time domain and secondly by signals in the frequency domain. The steps will be the same in both exercises but the length of the vectors will be different such as $\mathrm{N}$ in time domain and $\mathrm{N} / 2$ frequency domain. The steps considered in the construction of the reference state are defined below.

(i) Data collection: The vibration responses measured on the structure to be monitored are discretised into a vector. More than one measurement is considered having a total $\mathrm{M}$ vector signal realisations.

(ii) Representation of the signal vector: If the reference state wants to be computed in the time domain, the signal vectors are made of the discretised signal vectors of the measured signal with length $\mathrm{N}$ and ordered in columns within the data matrix $\mathbf{X}$ as shown in Equation 1. However, when the signal vectors used to construct the reference state are in the frequency domain representation, the signal vectors with length $\mathrm{N} / 2$ contain the amplitudes of the frequency spectra of measured vibration signals and ordered in columns within the matrix $\mathbf{Y}$ and shown in Equation 2.

$$
\begin{aligned}
& \mathbf{X}=\left(\begin{array}{llllll}
\mathbf{x}_{1} & \mathbf{x}_{2} & \ldots & \mathbf{x}_{\mathrm{m}} & \ldots & \mathbf{x}_{\mathrm{M}}
\end{array}\right) \\
& \mathbf{Y}=\left(\begin{array}{llllll}
\mathbf{y}_{1} & \mathbf{y}_{2} & \ldots & \mathbf{y}_{\mathrm{m}} & \ldots & \mathbf{y}_{\mathrm{M}}
\end{array}\right)
\end{aligned}
$$

(iii) Whether the reference estate is to be constructed in the time or in the frequency domain, this step will be applied in Equation 1 or Equation 2 respectively. This step is called embedding process. Each signal vector realisation is embedded into a matrix $\widetilde{\mathbf{X}}_{\mathrm{m}}$ or $\widetilde{\mathbf{Y}}_{\mathrm{m}}$ by $\mathrm{W}$-lagged copies of itself where $\mathrm{W}$ is the sliding window size and $\mathrm{m}=1 \ldots \mathrm{M}$ is the number of signal realisations. As the embedding process is applied to each signal vector realisation $\mathbf{x}_{\mathrm{m}}$ or $\mathbf{y}_{\mathrm{m}}$, the full embedded matrix will have a dimension $\mathrm{N} \times(\mathrm{M} \times \mathrm{W})$ or $\mathrm{N} / 2 \times(\mathrm{M} \times \mathrm{W})$ whether the signal vectors are in the time (see Equation 3 ) or in the frequency domain (see Equation 4).

$$
\begin{aligned}
& \widetilde{\mathbf{X}}=\left(\begin{array}{llllll}
\widetilde{\mathbf{X}}_{1} & \widetilde{\mathbf{X}}_{2} & \ldots & \widetilde{\mathbf{X}}_{\mathrm{m}} & \ldots & \widetilde{\mathbf{X}}_{\mathrm{M}}
\end{array}\right) \\
& \widetilde{\mathbf{Y}}=\left(\begin{array}{llllll}
\widetilde{\mathbf{Y}}_{1} & \widetilde{\mathbf{Y}}_{2} & \ldots & \widetilde{\mathbf{Y}}_{\mathrm{m}} & \ldots & \widetilde{\mathbf{Y}}_{\mathrm{M}}
\end{array}\right)
\end{aligned}
$$

(iv) The eigen-decomposition of the covariance matrix of the full embedded matrix is computed to obtain the eigenvalues and their corresponding eigenvectors ordered in decreasing order. The original signal vectors are then projected onto the eigenvectors to obtain the Principal Components (PCs).

(v) The last step is to define the Reference state. Finally, the Reconstructed Components (RCs) are obtained by convolving the Principal components with their associated eigenvector. Therefore, the reference state is constructed and represented by the matrix $\mathbf{R}$ that contains all Reconstructed Component in columns as shown in Equation 5.

$$
\mathbf{R}=\left(\begin{array}{llllll}
\mathbf{R}_{1} & \mathbf{R}_{2} & \cdots & \mathbf{R}_{\mathrm{m}} & \cdots & \mathbf{R}_{\mathrm{M}}
\end{array}\right)
$$

Therefore, the matrix $\mathbf{R}$ defines the reference state of the structure/system where the new observation signal vectors will be compared. Full details about the methodology are given in [5]. This manuscript only gives a short summary of the creation of the reference state step.

\section{Creation of the reference state in the time and in the frequency domain.}

In order to compare the effects on the creation of the reference state based on the time and frequency domain the same vibratory responses were considered in both analysis. The vibration signals utilised as input-data are represented in Fig. 1. Fig. 1(a) represents a free-decay 
vibration responses and Fig. 1(b) is its frequency spectrum representation.

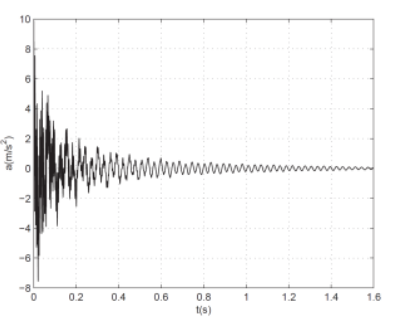

(a)

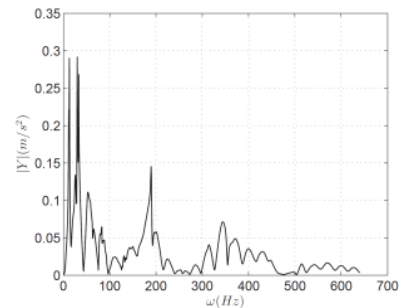

(b)
Fig. 1. Vibration signal. a) Free-decay acceleration signal and b) Frequency spectrum of the free-decay acceleration signal.

\subsection{Reference state based on vibration responses represented in the time domain}

This section studies the effect on the creation of the reference state based on the vibratory responses in the time domain representation. Seven signal realisations were considered $(M=7)$ for this analysis. Each signal realisation was discretised into a vector $\mathbf{x}_{\mathrm{m}}$ with a dimension of $\mathrm{N}=2048$ where the index m labels each realisation. The seven signal vectors were arranged in columns into the matrix $\mathbf{X}$ to define the data set considered for the construction of the reference state. The data set $\mathbf{X}$ is then processed by the steps described in the section 2. Two sliding window sizes were considered in the creation of the embedding matrix. One case uses a $\mathrm{W}=7$ and other case $\mathrm{W}=50$ to create the embedded matrix shown in Equation 3. The eigendecomposition of the covariance matrix of the full embedded matrix $\widetilde{\mathbf{X}}$ yields to a $(\mathrm{M} \mathrm{x} \mathrm{W})$ total number of eigenvalues with their corresponding eigenvectors, which more specifically are 49 for the case of $\mathrm{W}=7$ and 350 for the case of $\mathrm{W}=50$. As explained in section 2, the eigenvalues and eigenvectors are ordered in decreasing order by means of their variance content in the vibratory signal. Each eigenvalue defines the percentage of variance contained in the direction of its corresponding eigenvector. The percent of the partial variance of each eigenvalue in the total decomposition is calculated by Equation 6 where $\lambda_{\mathrm{k}}$ are the eigenvalues and $\mathrm{k}$ is the eigenvalue index which varies from $\mathrm{k}=1, \ldots$, $\mathrm{K}$ being $\mathrm{K}$ the total number of eigenvalues equal to ( $\mathrm{M} \mathrm{X}$ $\mathrm{W})$.

$$
\% \text { variance }=100 \times \frac{\lambda_{\mathrm{k}}}{\sum_{k=1}^{\mathrm{K}} \lambda_{\mathrm{k}}}
$$

Fig. 2 (a) and 2(b) represent the partial variance percent contained in each eigenvalue for the decomposition by using $\mathrm{W}=7$ and $\mathrm{W}=50$, respectively. These graphs represent the percentage variance only for 20 eigenvalues since the information contained in the rest of the eigenvalues is insignificant. Comparing the two graphs can be observed that for the decomposition with $\mathrm{W}=50$ the variance information contained in the first eigenvalues is smaller than for the case of $\mathrm{W}=7$. This behaviour stands in the fact that the information is distributed along the eigenvectors. As observed, for the case of $\mathrm{W}=50$ the number of eigenvalues increases and hence the variance percent contained in the first eigenvalues is reduced and distributed over all the components. However, for the case of $\mathrm{W}=7$ it can be observed that the variance percentage contained in the first eigenvalue is considerably larger than the rest of the eigenvalues.

The decomposition of the covariance matrix of the data set in eigenvalues and eigenvectors is to distribute the oscillatory components contained in the original signal vectors by means of their variance content. In this case, when the value of $\mathrm{W}$ is small, the variance contained in the first eigenvalues is large because the eigenvector corresponding to the first eigenvalue contains more than one oscillatory component and hence more variance of the original signal vector. On the other hand, when $\mathrm{W}$ is relatively large, the variance in the first eigenvalue reduces and hence less oscillatory components are contained in its corresponding eigenvector. In this case, it can be stated that large values of $\mathrm{W}$ will give better separable components in comparison with small values of W [7].
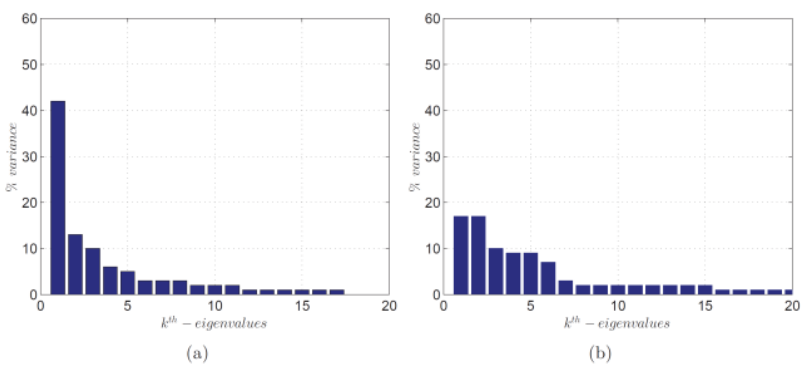

Fig. 2. Partial variance percent of each eigenvalue for a) $\mathrm{W}=7$ and b) $\mathrm{W}=50$ when the reference space is based in the time domain

Each RC is obtained based on the information contained in its corresponding PC weighted by its corresponding eigenvector. Each RC contains oscillatory components with the same content of variance in the vibratory response. The oscillatory components are less or more separated depending on the complexity of the vibratory system and also on the number of PCs obtained in the decomposition. In order to observe the oscillatory components contained in the RCs, the Fourier transform was applied to each RC separately of the matrix R. Fig. 3(a) and 3(b) represent the reconstructed signal using only the first two RCs (RC1 and RC2) for the case of W $=7$ and $\mathrm{W}=50$, respectively. Figures show the comparison of the reconstructed signal with the original signal to identify what oscillatory components are present in the reconstruction. In Fig. 3(a) can be observed that the two first RCs contain the oscillatory components for low frequencies up to $200 \mathrm{~Hz}$. It can be observed that the oscillatory components belonging to higher frequencies are not contained in these RCs. In a similar way, Fig. 3(b) shows the frequency spectrum of the reconstructed signal using the first two RCs for a W $=50$. It can be observed that only the oscillatory 
components belonging to the low frequencies up to 35 $\mathrm{Hz}$ are contained in the reconstructed signal. Also it can be observed that for the decomposition of the vibratory signal by using $\mathrm{W}=50$, the oscillatory components contained in the first RCs are very well separated in comparison with the decomposition by $\mathrm{W}=7$ where more oscillatory components are contained in the reconstructed signal.

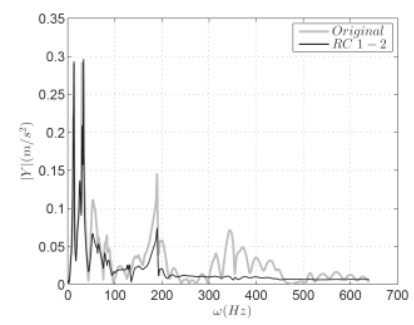

(a)

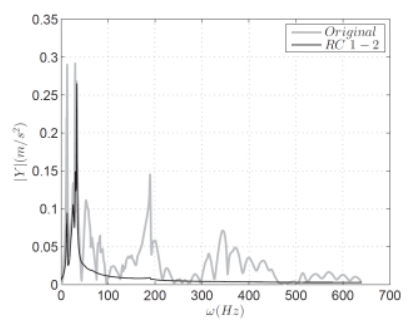

(b)
Fig. 3. Comparison between the original vibratory signal and the reconstructed signal using $2-\mathrm{RCs}$. The graphs show the effect of the methodology performed in the time domain. a) Comparison when $\mathrm{W}=7$ and b) $\mathrm{W}=50$

As explained above, when the decomposition is done by large values of $\mathrm{W}$ the oscillatory components are better separated. Therefore, the RCs have interpretable oscillatory components which can be used to identify particular modes. This can be beneficial when the interest is to extract particular modes of vibration.

\subsection{Reference state based on vibration responses represented in the frequency domain}

This section studies the effect on the creation of the reference state based on the vibratory responses in the frequency domain representation. Seven signal realisations were considered $(\mathrm{M}=7)$ for this analysis like in the previous section 3.1. Each signal realisation was discretised into a vector $\mathbf{y}_{\mathrm{m}}$ with a dimension of $\mathrm{N} / 2=1024$ where the index $\mathrm{m}$ labels each realisation. As explained above each signal vector represents the frequency domain representation of its corresponding $\mathbf{x}_{\mathrm{m}}$ vector.

The data set $\mathbf{Y}$ is then processed by the steps described in the section 2. Two sliding window sizes were also considered in the creation of the embedded matrix $\widetilde{\mathbf{Y}}$. One case uses a $\mathrm{W}=7$, the other case $\mathrm{W}=50$ to create the embedded matrix shown in Equation 4. The same sliding window sizes that in section 3.1 were considered in this analysis. The eigen-decomposition of the covariance matrix of the full embedded matrix $\widetilde{\mathbf{Y}}$ yields to 49 eigenvalues and eigenvectors for the case of $\mathrm{W}=7$ and 350 for the case of $\mathrm{W}=50$. In this way, the number of RCs obtained in the signal vectors decomposition will be the same that in section 3.1. The partial variance percent of each eigenvalue over the total eigenvalues was calculated by Equation 6 .
Fig 4(a) and 4(b) represent the partial variance percent contained in each eigenvalue for the decomposition by using $\mathrm{W}=7$ and $\mathrm{W}=50$, respectively. These graphs represent the percentage variance only for 20 eigenvalues since the information contained in the rest of the eigenvalues is insignificant. Comparing the two Figures, it can be observed that for the decomposition with $\mathrm{W}=50$, the variance information contained in the first eigenvalue is smaller than for $\mathrm{W}=7$. This behaviour stands in the fact that the information is distributed along the eigenvectors and when large values of $\mathrm{W}$ are considered, the number of eigenvectors increases, and hence the information is then distributed over all of them. As observed, for the case of $\mathrm{W}=50$ the number of eigenvalues increases with respect to the case of $\mathrm{W}=7$ and hence the variance percent contained in the first eigenvalues is reduced and distributed over all the rest of the eigenvalues. However, the variance percent contained in the first eigenvalue is the largest in both cases $(\mathrm{W}=7$ and $\mathrm{W}=50)$ with a significant difference in comparison with the other eigenvalues. Therefore, the first eigenvector contains the majority of the variance and it will have much more global contribution in the reconstruction of the signal vector. However, the rest of the eigenvectors have less variance content and their contribution in the reconstruction will be more local.

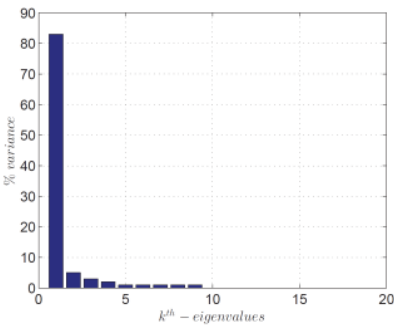

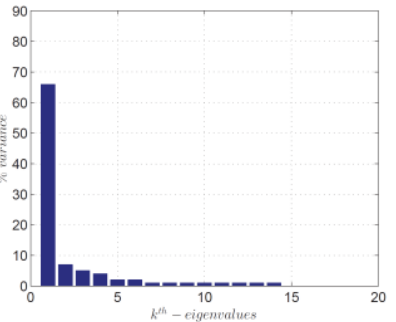

(b)
Fig. 4. Partial variance percent of each eigenvalue for a) $\mathrm{W}=7$ and b) $\mathrm{W}=50$ when the reference space is based in the frequency domain

Fig. 5(a) and 5(b) represent the reconstruction of the original signal vector by using only the first two RCs (RC1 and RC2) for the case of $\mathrm{W}=7$ and $\mathrm{W}=50$, respectively. In both graphs it can be observed that the reconstructed signal describes approximately the general trend of the original frequency spectrum. The estimated reconstructed signal contains information along the entire spectrum but it is more significant in the frequencies where the higher peaks are presented, in other words, at frequencies where the maximum of energy contained in the spectrum is concentrated. For the case of $\mathrm{W}=7$ (see Fig. 5(a)), the reconstructed signal is very good approximated and it contains well depicted peaks at low frequencies. However, for the case of $\mathrm{W}=$ 50 the reconstructed signal is smoother than in Figure 5(a), although the reconstruction still has a contribution to the entire spectrum (see Figure 5(b)). As mentioned above, the first eigenvectors contain the majority of the variability in terms of variance content and this is clearly represented in the reconstructed spectrum as observed in 
Figure 5(a) and 5(b) where a smooth trend is achieved by using only two RCs in the reconstruction. The selection of the sliding window size $\mathrm{W}$ is very important in the dimension of the decomposition and it depends on the goal of the study. Depending on what analysis is going to be addressed, the selection of $\mathrm{W}$ will help to obtain more understandable information [8].
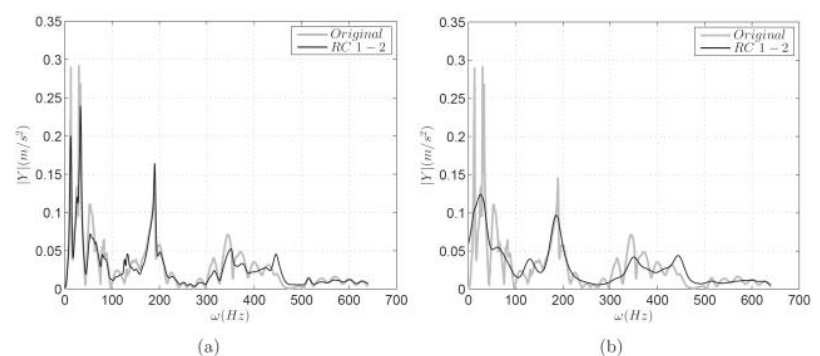

Fig. 5. Comparison between the original vibratory signal and the reconstructed signal using 2-RCs. The graphs show the effect of the methodology performed in the frequency domain. a) Comparison when $\mathrm{W}=7, \mathrm{~b}) \mathrm{W}=$ 50

\section{Discussions}

The analysis in sections 3.1 and 3.2 was to explain the effects when the reference state is constructed in the time or frequency domain. The comparison in the eigendecomposition and in the reconstruction was analysed. For both cases, it was observed that the first eigenvalues contain the majority of the variance and it decreases for the rest of the eigenvalues. In order to compare the eigen-decomposition for both, time and frequency domain, the eigenvalue spectra was calculated where the normalised eigenvalue and the normalised index are defined in Equation 7 respectively

$$
\log \left(\frac{\lambda_{\mathrm{k}}}{\sum_{k}^{\mathrm{K}} \lambda_{\mathrm{k}}}\right), k=\frac{k}{\mathrm{~K}}
$$

where $\lambda_{\mathrm{k}}$ is the $\mathrm{k}$-th eigenvalue. The results are represented in Fig. 6(a) and 6(b) when the decomposition, based on the time and frequency domain, is compared for the case of $\mathrm{W}=7$ and $\mathrm{W}=50$, respectively. For both sliding window size cases, it is observed that the first eigenvalues are smaller for the time domain case in comparison with the frequency domain case. The magnitude of eigenvalues decreases for both cases even though in the time domain case, the eigenvalues are larger than in frequency domain. This can explain that for the time domain case the difference in variance content between the first and the rest of the eigenvalues is less than for the frequency domain case. It is observed that the variance content is constantly distributed as shown in Fig. 6(a) for the time domain case, while a big jump can be observed between the first and the second eigenvalue for the case of the frequency domain. After this jump, the magnitude of the eigenvalues goes constantly down. In Fig. 6(b), it is observed that groups of eigenvalues are created in the time domain decomposition which means that they have similar amount of variance content. This proves that large values of $\mathrm{W}$ leads to more separable oscillatory components in the time domain decomposition. For the case of the frequency domain, the separation is clearly achieved between the first and the rest of the eigenvalues. In this case the effect on the selection of $\mathrm{W}$ will change the variance content in the first eigenvalue.
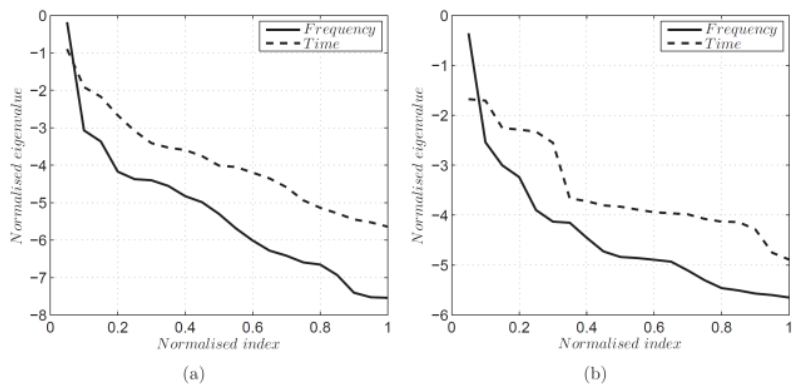

(b)

Fig. 6. Comparison of the eigenvalue spectra between time and frequency domain decomposition for a) $\mathrm{W}=7$ and b) $\mathrm{W}=50$

This means that when the frequency domain is used, the first eigenvector will contain the general information in the spectrum which leads to a smooth trend in the reconstruction as shown in Fig. 5. Then, all frequency modes captured in the original frequency domain are also included in the reconstructed spectrum. The rest of the eigenvectors will add the fluctuations on the spectral line described by the first RC. It is true that the reconstructed spectrum does not have the same amplitude as the original signal vector but it still conserves the general weight of distribution in the entire spectrum.

In the case of the time domain, the behaviour observed in the reconstruction has a different interpretation than in the case of the frequency domain. The decomposition in the time domain leads to a number of oscillatory components which can be interpreted separately. As shown in Fig. 3 only a few frequency bands are included in the reconstruction signal. The separability of these oscillatory components depends on the value of sliding window size. As a general rule, large values of $\mathrm{W}$ will give more separated oscillatory components. The benefit of obtaining separated oscillatory components is to identify the particular modes which can contain relevant information.

With the comparison of the creation of the reference state based on time or frequency domain developed in the above sections, it can be concluded that the selection of one of these analysis depends on the case to study. When a time domain representation is used in the methodology, separated oscillatory components are obtained. The information contained in the reconstructed signal will depend on which oscillatory components are considered in the reconstruction. The separability of 
these oscillatory components will depend on the complexity of the vibratory system and also on the dimension of decomposition defined by $\mathrm{W}$. This analysis can be beneficial when a predominant mode of vibration is to be analysed and therefore the methodology can be useful for mode extraction or identification. However, when the frequency domain representation is used, it can be observed that a smoother version of the original spectrum is obtained by the reconstructed spectrum. The reconstructed spectrum is more approximated to the original one, when more RCs are considered in the reconstruction. This analysis can be beneficial when more than one vibration mode is considered in the analysis.

As the reconstruction describes the general trend of the spectral line, all rotational patterns are considered in the reconstructed spectrum with small number of RCs.

\section{Conclusions}

In this work is studied a stage of a methodology based on Singular Spectrum Analysis which decomposes the vibration responses in a certain number of principal components having in consideration all rotational patterns at any frequency. One of the steps of the methodology is to create a reference state where the observations can be compared for damage assessment. The data used to create the reference state determines how the information is represented in the reference state and therefore how meaningful and informative are the feature vectors for damage assessment. The study presents the effects on the creation of the reference state when the data is introduced in the time or frequency domain and the main conclusions are summarised below.

When the reference state is constructed in the time domain representation, the reference state provides separated and interpretable oscillatory components distributed in the reconstructed components. If the aim is to have information about all the oscillatory components presented in the vibratory response more reconstructive components should be retained in order to reconstruct an approximated original vibration response. As the reconstructed components are separated, the selection of particular oscillatory components can depend on the application.

On the other hand, when the reference state is created in the frequency domain representation, the first reconstructed component describes the general trend of the original spectral line of the frequency spectrum. The rest of the reconstructed components represent the fluctuations along the spectral line. Therefore, the reconstructed spectrum with only the first RC gives more weight to the frequencies bands where the maximum energy is concentrated by means of the amplitudes/peaks at different frequencies. All the rotational patterns are considered with only the first reconstructive component.

\section{References}

1. C. P. Fritzen, Key Eng. Mat. 293, 3-20 (2005)
2. G. Gui, H. Pan, Z. Lin, Y. Li, and Z. Yuan, KSCE J. Civil Eng. 21, 523-534 (2017)

3. C. R. Farrar, k. Worden, Phil. Trans. R. Soc. A 365, 303-315 (2007)

4. K. Worden, C.R Farrar, G. Manson and G. Park, Phil. Trans. R. Soc. A 463, 1639-1664 (2007).

5. D. Garcia, and I. Trendafilova, J. Sound and Vib. 333, 7036-7050 (2014).

6. N. Golyandina, and A. Zhigljavsky, Singular Spectrum Analysis for Time Series (Springer briefs in statistics, 2013)

7. M. C. R. Leles, A. S. V. Cardoso, M. G. Moreira, H. N. Guimares, C. M. Silva, and A. Pitsillides, IEEE International Symposium on Signal Processing and Information Technology (2016).

8. J. B. Rafert, J. Zabalza, S. Marshall, and J. Ren, App. Spectrosc. 70 1528-1588 (2016). 